\title{
AXISYMMETRIC FE MODEL TO ANALYSIS OF THERMAL STRESSES IN A BRAKE DISK
}

\author{
Adam Adamowicz \\ Bialystok University of Technology (BUT), Faculty of Mechanical Engineering, Bialystok, Poland \\ e-mail: a.adamowicz@pb.edu.pl
}

\begin{abstract}
The preceding to this study was a finite element analysis (FEA) of transient temperature fields in a disk brake. In this paper, a FE model to determine corresponding quasi-static stresses in a such tribosystem is proposed. Numerical simulation of a single braking process for the 2D axisymmetric model of the brake disk is carried out. It is assumed that the contact pressure on the friction surfaces is constant, and the angular speed decreases linearly. Evolutions and spatial distributions of the components of the stress tensor and the equivalent Huber-Mises stress in the disk are analyzed.
\end{abstract}

Keywords: frictional heating, thermal stresses, pad-disk brake system, finite element method

\section{Nomenclature}

B, C, D - matrix of differential operators, heat capacity matrix and elasticity matrix

$c, h \quad-$ specific heat $[\mathrm{J} /(\mathrm{kg} \mathrm{K})]$ and heat transfer coefficient $\left[\mathrm{W} /\left(\mathrm{m}^{2} \mathrm{~K}\right)\right]$

$E \quad-$ Young's modulus [MPa]

$f \quad-$ coefficient of friction

F $\quad-\quad$ nodal force vector due to initial strain

$J_{2} \quad-$ second invariant of the deviatoric stress tensor $\left[\mathrm{MPa}^{2}\right]$

$k, K \quad-$ thermal diffusivity $\left[\mathrm{m}^{2} / \mathrm{s}\right]$ and thermal conductivity $[\mathrm{W} /(\mathrm{m} \mathrm{K})]$

$\mathbf{K}, \mathbf{H}_{T} \quad-$ stiffness matrix and conductivity matrix

$p_{0} \quad-$ contact pressure $[\mathrm{MPa}]$

$q \quad-$ heat flux density $\left[\mathrm{W} / \mathrm{m}^{2}\right]$

Q $\quad-$ vector of applied thermal load

$r, R \quad-\quad$ inner and outer radius, respectively $[\mathrm{m}]$

$t, t_{s} \quad-$ time and braking time $[\mathrm{s}]$

$T, T_{a}, T_{0}-$ temperature, ambient temperature and initial temperature $\left[{ }^{\circ} \mathrm{C}\right]$

$\mathbf{T} \quad-$ temperature vector

$\mathbf{u} \quad-$ column vector including components of displacements of the point

$u_{r}, u_{z} \quad-$ displacement components in radial $r$ and axial $z$ direction, respectively $[\mathrm{m}]$

$z \quad-$ axial coordinate

Greek symbols

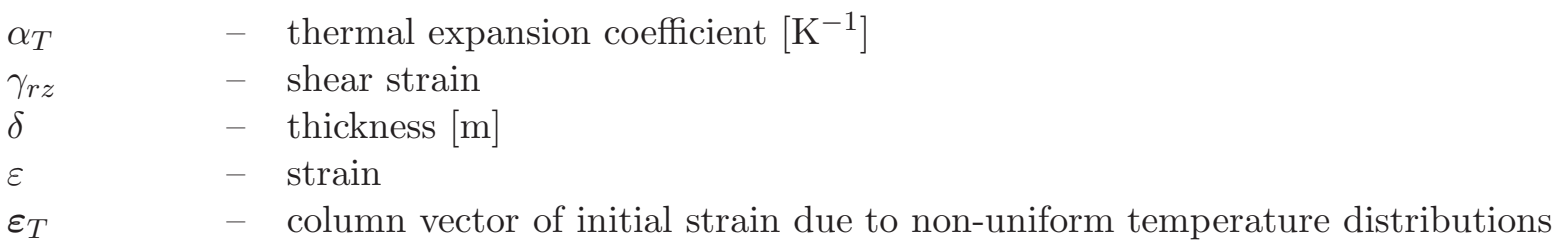




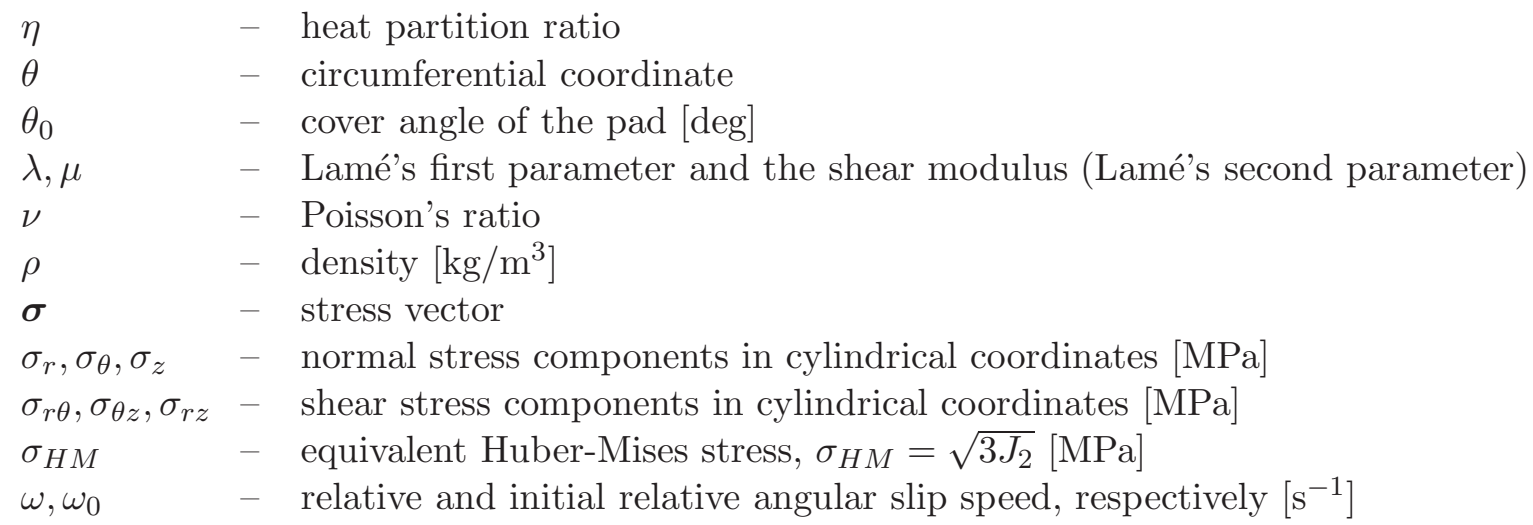

Subscripts

$d$ - indicates disk, $p$ - indicates pad

\section{Introduction}

The basis of analysis of problems of heating in brake systems and the concurrent phenomena are solutions of thermal problems of friction - equations of motion, boundary-value heat conduction problems and laws of wear. These solutions may be obtained by means of analytical methods (e.g. Evtushenko et al., 2005, Matysiak et al., 2007) or several numerical and analytical-numerical methods, among which the most popular is the finite element method (Zienkiewicz et al., 2005) and the boundary element method (e.g. Burczyński, 1995). In this paper, results of calculations carried out using finite element software package MSC.Nastran and personal software are presented.

During braking stationary pads are pressed to the rubbing path of the rotating disk and mechanical energy is converted into heat. Temperature on the contact surfaces rises rapidly, which results in temperature gradients and stresses due to thermal expansion. Interactions between the temperature and the stresses are coupled. In calculations, however, this relationship is frequently omitted due to small deformations, analyzing the heat conduction and the thermoelasticity problems separately (Zagrodzki, 1985; Dufrénoy and Weichert, 2003; Collignon et al., 2013).

Both the disk and pads, are exposed to various negative effects resulting from high temperature, however, the essence of the material failure of both components is different and requires separate considerations. In typical applications of disk brakes, the pads cover the working surface of the disk partly, therefore the temperature in the circumferential direction is non-uniform. A comparative analysis of axisymmetric and three-dimensional transient temperature fields in a brake disk was carried out by Adamowicz and Grzes (2011a,b). It was observed that the temperature change during braking for a particular location on the contact surface calculated using the three-dimensional FE model revealed apparent fluctuations according to the pad-disk relative displacement, whereas the axisymmetric model allowed one to calculate average contact temperature changes. Therefore, an effect of non-uniform heating of the friction surface is frequently neglected above a certain relative sliding speed or alternately if the cover angle of the pads is close to $2 \pi$ (Yevtushenko and Grzes, 2011a,b; 2012). A three-dimensional model to study an influence of convective cooling on the temperature of the disk during a single and a multiple braking process was developed by Adamowicz and Grzes (2011a,b) based on the model from the article by Adamowicz and Grzes (2011a,b). Further investigations of 3D temperature field of the disk concerned analysis at temperature-dependent thermophysical properties of materials (Adamowicz and Grzes, 2013). A review paper on numerical modeling of the frictional heat generation phenomenon in disk brakes and clutches using the finite element method was given by Yevtushenko and Grzes (2010). 
Axisymmetric temperature and quasi-static thermal stress fields in the disks of a wet clutch were studied in articles by Zagrodzki $(1985,1990)$. Two different types of the disks arranged alternately: the disk with a steel core coated with the friction material and the disk made entirely of steel were analyzed using the finite difference method (FDM) and the finite element method.

A thermoelastic instability phenomenon for an axisymmetric model of a pad-disk brake system during braking was studied by Choi and Lee (2003, 2004). Normal stress changes during braking were calculated and analyzed.

The thermal cracking mechanism of brake disks was studied in the article by Mackin et al. (2002). At the first stage, forces acting on the friction elements during braking were determined. Then braking conditions (braking time, initial speed, number of brake applications) that may lead to the development of thermal cracks were established. Based on the calculated radial, circumferential and Huber-Mises equivalent stresses as well as the Coffin-Manson formula, the number of cycles to the appearance of damage to the brake disk was predicted.

An axisymmetric FE model of a high speed train disk brake made of steel to analyze the fracture mechanisms based on experimental studies was developed in the article by Sakamoto and Hirakawa (2005). The comparative analysis of circumferential stress distributions obtained from numerical calculations and experimental measurements using the strain gauges was carried out. An extensive discussion to improve the strength of the disk by varying the chemical composition of the material was carried out.

A thermomechanical analysis of the transient temperature field and the stress in the pad-disk brake system during a single braking was carried out in the article by Gao et al. (2007). The calculations were conducted using FE based software ANSYS 8.1. An algorithm for thermomechanical coupling for the contact problem was developed by means of ANSYS Parametric Design Language (APDL). An emphasis was placed on the discussion of the thermal fatigue question leading to thermal cracks.

A coupled thermomechanical problem for a multi-plate dry clutch made of a composite material was studied by Zhao et al. (2008). Computations of the transient temperature field and normal stress for a single clutch engagement and the disengagement using an axisymmetric contact FE model were carried out. The pressure and temperature distributions in the radial direction as well as the normal radial, circumferential and tangential stress changes were analyzed.

A detailed review of studies concerning FE modeling of thermal stresses in disk brakes and clutches was given in the article by Yevtushenko et al. (2014).

Temperature distributions, equivalent Huber-Mises stresses and strains in a mine hoist brake disc were studied by article Scieszka and Zolnierz (2014). The calculations were carried out both for a solid and divided disc (2, 4 and 8 sections) using the 3D thermomechanical finite element contact model. The model was validated by infrared measurements of the temperature field in real industrial conditions.

In this paper, axisymmetric quasi-static thermal stresses in a cast-iron (ChNMKh) brake disk are analyzed using the finite element method. It should be noted, however, that the conclusions drawn are also valid for other materials whose physical and mechanical properties are similar.

\section{Statement of the problem}

Consider a single braking process from the initial angular speed $\omega_{0}$ at time $t=t_{0}$ to standstill in the pad-disk brake system shown in Fig. 1a. The displacement of the pads induces contact pressure on the working surfaces of the sliding components, and the angular speed due to friction decreases linearly according to the relationship

$$
\omega(t)=\omega_{0}\left(1-t / t_{s}\right) \quad 0 \leqslant t \leqslant t_{s}
$$


The contact pressure is constant and uniformly distributed $p=p_{0}$ on the friction surfaces. The slipping process is accompanied by heat generation. The heat flow takes place in the disk and the pads. In view of relatively short duration of the process, the temperature on the inner cylindrical surface of the disk is negligible, therefore the computational model is restricted only to the disk annular section symmetrical relative to its mid-plane ('hat section' is neglected). The pads in calculations are not included.

The non-uniform temperature distribution in the brake disk induces thermal stresses due to thermal expansion. It is assumed that:

- the material of the disk is uniform, isotropic, and its physical and mechanical properties are temperature independent;

- the heating process is described by the linear theory of heat conduction (parabolic type). Since heat transfer through convection and thermal radiation is negligibly small during single braking (Adamowicz and Grzes, 2011), in this study it is not taken into account;

- the corresponding thermal stresses are determined from the solution of the linear system of equations of uncoupled thermoelasticity;

- due to geometrical and load symmetry of the disk about the mid-plane, the computational region is restricted to the half $\delta_{d}$ of its thickness;

- mechanical interactions are neglected due to their minor impact on the resulting load of the pads and the disk (Dufrénoy and Weichert, 2003).

Based on the abovementioned assumptions, the corresponding boundary value problem of heat conduction and boundary problem of thermoelasticity will be formulated.

(a)

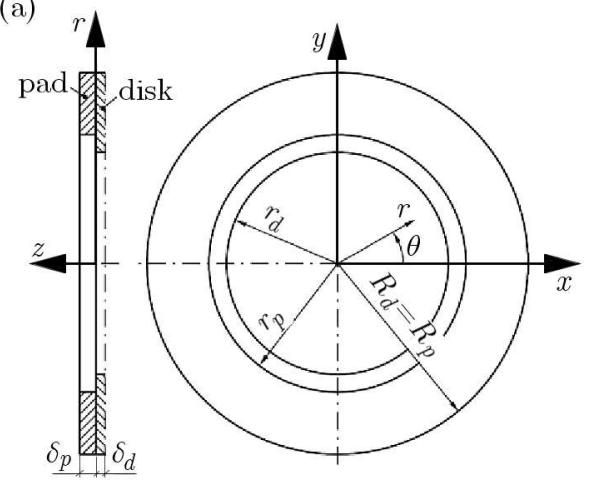

(b) heat conduction problem

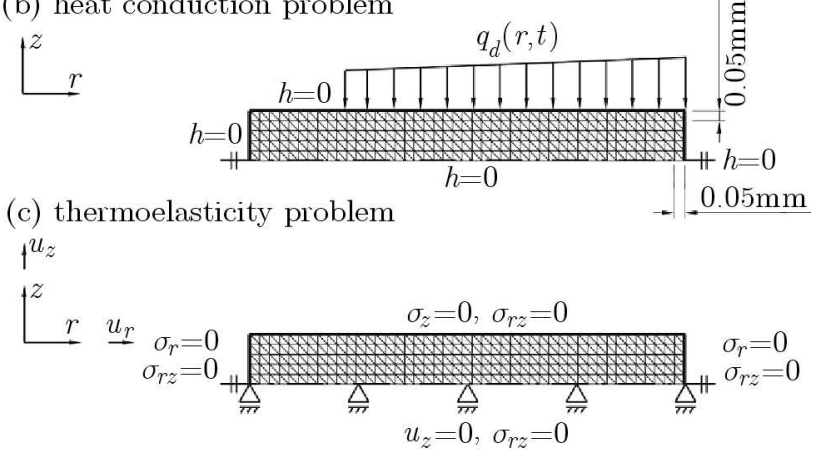

Fig. 1. A schematic of the pad-disc brake system (a) and the FE mesh with the boundary conditions for the heat conduction problem (b) and thermoelasticity problem (c)

\subsection{Heat conduction problem}

The transient temperature field $T(r, z, t)$ of the disk will be determined from the solution of the heat conduction equation of parabolic type (Fig. 1b)

$$
\frac{\partial^{2} T}{\partial r^{2}}+\frac{1}{r} \frac{\partial T}{\partial r}+\frac{\partial^{2} T}{\partial z^{2}}=\frac{1}{k_{d}} \frac{\partial T}{\partial t} \quad r_{d} \leqslant r \leqslant R_{d} \quad-\delta_{d} \leqslant z \leqslant 0
$$

at the following boundary conditions (Fig. 1b)

$$
\begin{aligned}
& \left.K_{d} \frac{\partial T}{\partial z}\right|_{z=0}=q_{d}(r, t) \quad r_{p} \leqslant r \leqslant R_{p} \quad 0 \leqslant t \leqslant t_{s} \\
& \left.\frac{\partial T}{\partial z}\right|_{z=0}=0 \quad r_{d} \leqslant r \leqslant r_{p} \quad 0 \leqslant t \leqslant t_{s}
\end{aligned}
$$


and

$$
\begin{array}{lcc}
\left.\frac{\partial T}{\partial r}\right|_{r=R_{d}}=0 & -\delta_{d} \leqslant z \leqslant 0 & 0 \leqslant t \leqslant t_{s} \\
\left.\frac{\partial T}{\partial r}\right|_{r=r_{d}}=0 & -\delta_{d} \leqslant z \leqslant 0 & 0 \leqslant t \leqslant t_{s} \\
\left.\frac{\partial T}{\partial z}\right|_{z=-\delta_{d}}=0 & r_{d} \leqslant r \leqslant R_{d} & 0 \leqslant t \leqslant t_{s}
\end{array}
$$

where

$$
\begin{aligned}
& q_{d}(r, t)=\eta f \omega(t) r p_{0} \quad r_{p} \leqslant r \leqslant R_{p} \quad 0 \leqslant t \leqslant t_{s} \\
& q_{p}(r, t)=(1-\eta) f \omega(t) r p_{0} \quad r_{p} \leqslant r \leqslant R_{p} \quad 0 \leqslant t \leqslant t_{s} \\
& \eta=\frac{\sqrt{K_{d} \rho_{d} c_{d}}}{\sqrt{K_{d} \rho_{d} c_{d}}+\sqrt{K_{p} \rho_{p} c_{p}}}
\end{aligned}
$$

At $t=0$ the temperature of the disk is constant and equals $T_{0}$.

\subsection{Thermoelasticity problem}

The non-uniform temperature field of the disk induces thermal stresses. That effect is particularly evident for the friction pairs subjected to high temperature gradients, which occur during braking (Chichinadze, 1967).

The equilibrium at any point of the area in question satisfies the Navier-Lamé differential equations of equilibrium (Noda et al., 2003)

$$
\begin{aligned}
& (2 \mu+\lambda)\left(\frac{\partial^{2} u_{r}}{\partial r^{2}}+\frac{1}{r} \frac{\partial u_{r}}{\partial r}-\frac{u_{r}}{r^{2}}\right)+\mu \frac{\partial^{2} u_{r}}{\partial z^{2}}+(\mu+\lambda) \frac{\partial^{2} u_{z}}{\partial z \partial r}=\alpha_{T} \frac{\partial T}{\partial r} \\
& \mu\left(\frac{\partial^{2} u_{z}}{\partial r^{2}}+\frac{1}{r} \frac{\partial u_{z}}{\partial r}\right)+(2 \mu+\lambda) \frac{\partial^{2} u_{z}}{\partial z^{2}}+(\mu+\lambda)\left(\frac{\partial^{2} u_{r}}{\partial r \partial z}+\frac{1}{r} \frac{\partial u_{r}}{\partial z}\right)=\alpha_{T} \frac{\partial T}{\partial z}
\end{aligned}
$$

The system of partial differential equations (2.10) will be solved at the following boundary conditions (Fig. 1c):

- on the friction surface $z=0$

$$
\sigma_{z}=0 \quad \sigma_{r z}=0 \quad r_{d} \leqslant r \leqslant R_{d}
$$

- on the plane of symmetry $z=-\delta_{d}$

$$
u_{z}=0 \quad \sigma_{r z}=0 \quad r_{d} \leqslant r \leqslant R_{d}
$$

- on the inner cylindrical surface of the disk $r=r_{d}$

$$
\sigma_{r}=0 \quad \sigma_{r z}=0 \quad-\delta_{d} \leqslant z \leqslant 0
$$

- on the outer cylindrical surface of the disk $r=R_{d}$

$$
\sigma_{r}=0 \quad \sigma_{r z}=0 \quad-\delta_{d} \leqslant z \leqslant 0
$$

where (Noda et al., 2003)

$$
\begin{aligned}
& \sigma_{r}=(2 \mu+\lambda) \varepsilon_{r}+\lambda\left(\varepsilon_{\theta}+\varepsilon_{z}\right)-\alpha_{T}\left(T-T_{0}\right) \\
& \sigma_{\theta}=(2 \mu+\lambda) \varepsilon_{\theta}+\lambda\left(\varepsilon_{r}+\varepsilon_{z}\right)-\alpha_{T}\left(T-T_{0}\right) \\
& \sigma_{z}=(2 \mu+\lambda) \varepsilon_{z}+\lambda\left(\varepsilon_{r}+\varepsilon_{\theta}\right)-\alpha_{T}\left(T-T_{0}\right) \\
& \sigma_{r z}=\mu \gamma_{r z}
\end{aligned}
$$

and

$$
\varepsilon_{r}=\frac{\partial u_{r}}{\partial r} \quad \varepsilon_{\theta}=\frac{u_{r}}{r} \quad \varepsilon_{z}=\frac{\partial u_{z}}{\partial z} \quad \gamma_{r z}=\frac{\partial u_{r}}{\partial z}+\frac{\partial u_{z}}{\partial r}
$$




\section{FE discretization}

Heat conduction problem. In this Section, a general finite element procedure to obtain an approximate solution to the thermal problem will be given. Applying Galerkin's method to parabolic heat conduction equation (2.2), we get the following matrix equation (Noda et al., 2003)

$$
\mathbf{C} \frac{d \mathbf{T}}{d t}+\mathbf{H}_{T} \mathbf{T}=\mathbf{Q}
$$

The solution of Eq. (3.1) relies on the time integration methods. The following finite difference approximations are introduced

$$
\frac{1}{\Delta t}\left(\{\mathbf{T}\}_{t+\Delta t}-\{\mathbf{T}\}_{t}\right)=(1-\beta)\left\{\frac{d \mathbf{T}}{d t}\right\}_{t}+\beta\left\{\frac{d \mathbf{T}}{d t}\right\}_{t+\Delta t}
$$

where $\{\mathbf{T}\}_{t}$ is the temperature at time $t$. Substituting Eq. (3.2) to Eq. (3.1), the system of linear algebraic equations is obtained

$$
\left(\mathbf{C}+\beta \Delta t \mathbf{H}_{T}\right)\{\mathbf{T}\}_{t+\Delta t}=\left[\mathbf{C}-(1-\beta) \mathbf{H}_{T} \Delta t\right)\{\mathbf{T}\}_{t}+(1-\beta) \Delta t\{\mathbf{Q}\}_{t}+\beta \Delta t\{\mathbf{Q}\}_{t+\Delta t}
$$

In Eq. (3.3) all of the terms on the right-hand side are known. The only unknown is the temperature $\{\mathbf{T}\}_{t+\Delta t}$ at the time moment $t+\Delta t$. The value of the parameter $\beta$ may vary from 0 to 1 , defining the particular algorithm to be used. If $\beta=0$, the algorithm is the forward difference method (Euler method), if $\beta=0.5$, the algorithm is the Crank-Nicolson method, and if $\beta=1$, the algorithm is the backward difference method. If $\beta \geqslant 0.5$, the algorithm is unconditionally stable. For $\beta<0.5$, the algorithm is conditionally stable and depends on some critical time step (Huebner and Thornton, 1982).

\subsection{Thermoelasticity problem}

The constitutive equation (a relationship between the strain and the stress) for the elastic body including initial strain $\varepsilon_{T}$, resulting from thermal expansion (or other cause) may be written in the following matrix notation (Zienkiewicz et al., 2005)

$$
\boldsymbol{\sigma}=\mathbf{D}\left(\varepsilon-\varepsilon_{T}\right)=\mathbf{D B u}-\mathbf{D} \varepsilon_{T}
$$

where $\boldsymbol{\sigma}=\left[\sigma_{r}, \sigma_{\theta}, \sigma_{z}, \sigma_{r z}\right]^{\mathrm{T}}$.

The elasticity matrix $\mathbf{D}$ contains appropriate material properties (elastic constants) and is obtained from the relationship between stresses and strains)

$$
\mathbf{D}=\frac{E}{(1+\nu)(1-2 \nu)}\left[\begin{array}{cccc}
1-\nu & \nu & \nu & 0 \\
\nu & 1-\nu & \nu & 0 \\
\nu & \nu & 1-\nu & 0 \\
0 & 0 & 0 & \frac{1-2 \nu}{2}
\end{array}\right]
$$

The strain-displacement relations of the elastic body (brake disk) in the matrix form are as follows

$$
\varepsilon=\mathbf{B u}
$$

where $\boldsymbol{\varepsilon}=\left[\varepsilon_{r}, \varepsilon_{\theta}, \varepsilon_{z}, \gamma_{r z}\right]^{\mathrm{T}}, \mathbf{u}=\left[u_{r}, u_{z}\right]^{\mathrm{T}}$ - represents the column vector including components of displacement of the point, of the $r$ and $z$ of the cylindrical system coordinate system; $\mathbf{B}$ is the matrix of differential operators

$$
\mathbf{B}=\left[\begin{array}{cccc}
\frac{\partial}{\partial r} & \frac{1}{r} & 0 & \frac{\partial}{\partial z} \\
0 & 0 & \frac{\partial}{\partial z} & \frac{\partial}{\partial r}
\end{array}\right]^{\mathrm{T}}
$$


For the isotropic material vector of components of the tensor of thermal strain $\varepsilon_{T}$ is given by

$$
\varepsilon_{T}=\alpha_{T} T[1,1,1,0]^{\mathrm{T}}
$$

where $T$ is an average increase in temperature in the finite element.

In mechanical problems, it is convenient to use the variational principle of minimization of functional of total potential energy, which, as a result, leads to the system of linear algebraic equations

$$
\mathbf{K u}=\mathbf{F}
$$

The stiffness matrix of an element $\mathbf{K}$ and the force vector $\mathbf{F}$ due to initial strain (non-uniform temperature distribution) is given by

$$
\mathbf{K}=2 \pi \iint_{A} \mathbf{B}^{\mathrm{T}} \mathbf{D} \mathbf{B} r d r d z \quad \mathbf{F}=2 \pi \iint_{A} \mathbf{B}^{\mathrm{T}} \mathbf{D} \varepsilon_{0} r d r d z
$$

The integrals in equations (3.10) are taken over the whole surface area $A$ on which the tractions take place (Zienkiewicz et al., 2005).

The heat conduction and the thermoelasticity problems are analyzed in two stages using the finite element based software MSC.Patran/MSC.Nastran (MSC.Nastran Thermal Analysis User's Guide, 2002). First the numerical solution of heat conduction problem during braking allows one to determine the transient temperature field for the established time steps. Then the obtained outcomes are used as the boundary conditions (non-uniform temperature distribution) for the thermoelasticity problem result in the stress field for the specific time moments. Due to the large number of time steps and consequently great amount of data for further transformation, authorial software automatizing the process of generation of computational cases to study is developed. The pre-/post processor (MSC.Patran) is used for the realization of operations based on accessing the data from the heat conduction problem and generation of the reports, which are then transformed to the form of the boundary conditions for the thermoelasticity problem. The script written in Python programming language generates the input files to the MSC.Nastran. For the presentation of the significant number of results in the form of the stress tensor components changes during braking, an additional program is developed.

According to the abovementioned algorithm, at the first stage of the computations, the transient temperature fields of the disk with the fixed time step of the analysis $\Delta t=0.001 \mathrm{~s}$ are calculated. Further evaluation of quasi-static thermal stresses due to non-uniform temperature fields requires consideration of separate computational cases for the established time moments. In order to obtain smooth thermal stress changes in the disk, time step $\Delta t=0.01 \mathrm{~s}$ is established.

In order to analyze the thermal and mechanical problem separately, the same FE element mesh is used. The region of the disk is divided into 209000 linear triangular three-node axisymmetric elements 'CTRIAX6' and 105561 nodes. The dimension of each element in the axial and radial direction is approximately equal $(\Delta r=\Delta z=0.05 \mathrm{~mm})$ (Fig. 1b).

\section{Numerical analysis}

A single braking process at a constant deceleration from the initial angular speed of the disk $\omega_{0}=88.464 \mathrm{~s}^{-1}$ to standstill during $t=t_{s}=3.96 \mathrm{~s}$ is studied. It is assumed that at the initial time moment of the braking process, the temperature of the disk is constant and equal to the ambient temperature $T_{0}=T_{a}=20^{\circ} \mathrm{C}$. The dimensions and properties of the components of the pad-disk brake system and the operating parameters of the process are given in Table 1. 
Table 1. Dimensions (Talati and Jalalifar, 2009), properties of materials (Chichinadze, 1967) of the pad and the disk and operating parameters

\begin{tabular}{|l|c|c|}
\hline \multicolumn{1}{|c|}{ Parameter } & Disk (cast iron ChNMKh) & Pad (FMC-11) \\
\hline \hline inner radius, $r[\mathrm{~mm}]$ & 66 & 76.5 \\
\hline outer radius, $R[\mathrm{~mm}]$ & 5.5 & 113.5 \\
\hline thickness, $\delta[\mathrm{mm}]$ & 51 & 64.5 \\
\hline pad arc length, $\theta_{0}[\mathrm{deg}]$ & 7100 & 34.3 \\
\hline thermal conductivity, $K[\mathrm{~W} /(\mathrm{mK})]$ & 1.44 & 1700 \\
\hline density, $\rho\left[\mathrm{kg} / \mathrm{m}^{3}\right]$ & 99.97 & \\
\hline thermal diffusivity, $k \cdot 10^{5}\left[\mathrm{~m}^{2} / \mathrm{s}\right]$ & 0.29 \\
\hline Young's modulus, $E[\mathrm{GPa}]$ & $1.08 \cdot 10^{-5}$ & \\
\hline Poisson's ratio, $\nu[-]$ & \multicolumn{2}{|c|}{88.464} \\
\hline thermal expansion coefficient, $\alpha_{T}\left[\mathrm{~K}^{-1}\right]$ & \multicolumn{2}{|c|}{3.96} \\
\hline initial angular speed of the disk, $\omega_{0}\left[\mathrm{~s}^{-1}\right]$ & \multicolumn{2}{|c|}{1.47} \\
\hline braking time, $t_{s}[\mathrm{~s}]$ & \multicolumn{2}{|c|}{0.5} \\
\hline contact pressure, $p_{0}[\mathrm{MPa}]$ & 20 \\
\hline coefficient of friction, $f$ & 20 \\
\hline initial temperature, $T_{0}\left[{ }^{\circ} \mathrm{C}\right]$ & \multicolumn{2}{|c|}{} \\
\hline ambient temperature, $T_{a}\left[{ }^{\circ} \mathrm{C}\right]$ & \multicolumn{2}{|c|}{} \\
\hline
\end{tabular}

(a)

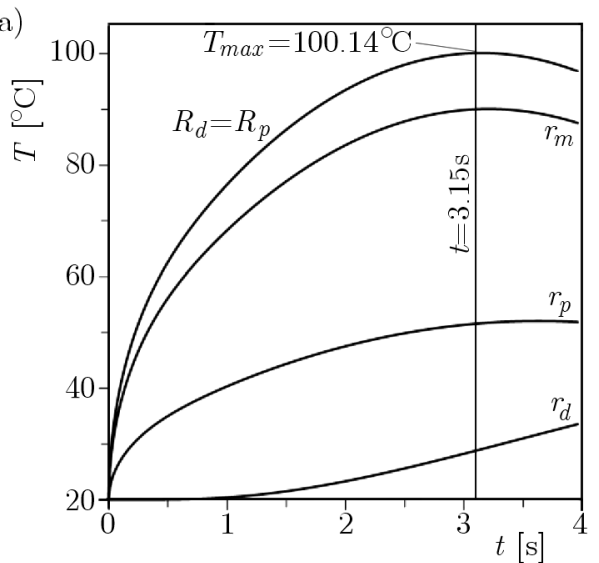

(c)

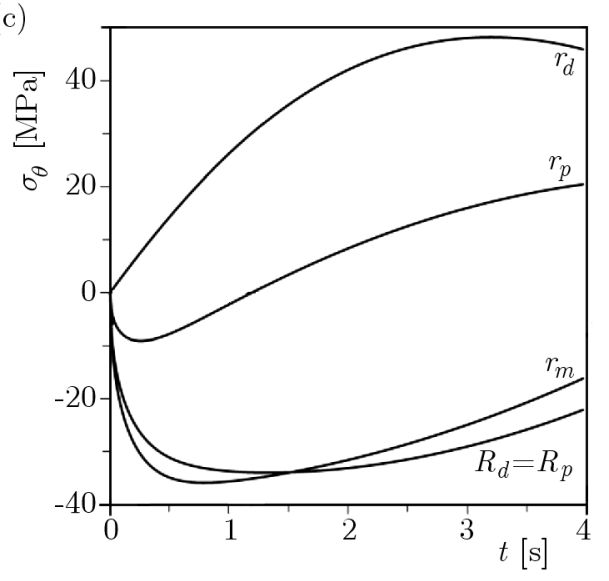

(b)

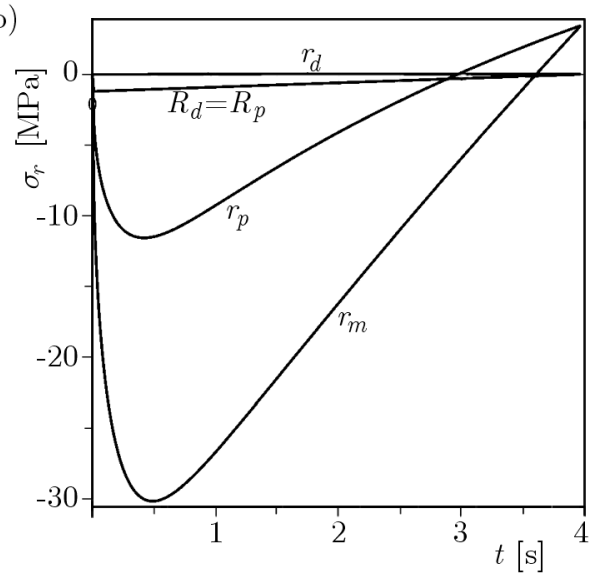

(d)

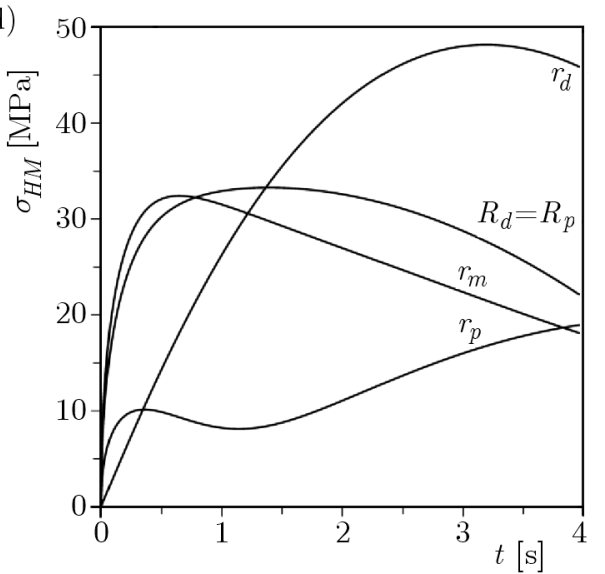

Fig. 2. Temperature changes on the contact surface of the disk $(z=0)$ during braking (a), radial (b), circumferential (c), and equivalent Huber-Mises (d) stress changes on the contact surface of the disk $(z=0)$ during braking 
As mentioned, at the first stage the transient temperature distributions in the disk during braking are calculated. The values of temperatures obtained at the given time moments, with a suitably selected time step, are used for calculation of the distribution of quasi-static thermal stresses.

The temperature changes on the friction surface $z=0$ during braking for specific radii $\left(r_{d}=66 \mathrm{~mm}\right.$ - inner radius of the disk, $r_{p}=76.5 \mathrm{~mm}$ - inner radius of the pad, $r_{m}=95 \mathrm{~mm}-$ average radius of the pad, and $R_{p}=R_{d}=113.5 \mathrm{~mm}$ - outer radius of the pad and the disk) are shown in Fig. 2a.

(a)

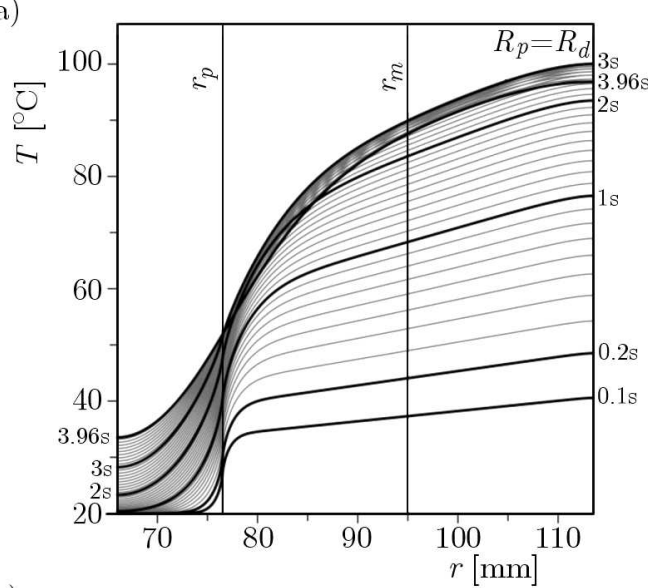

(c)

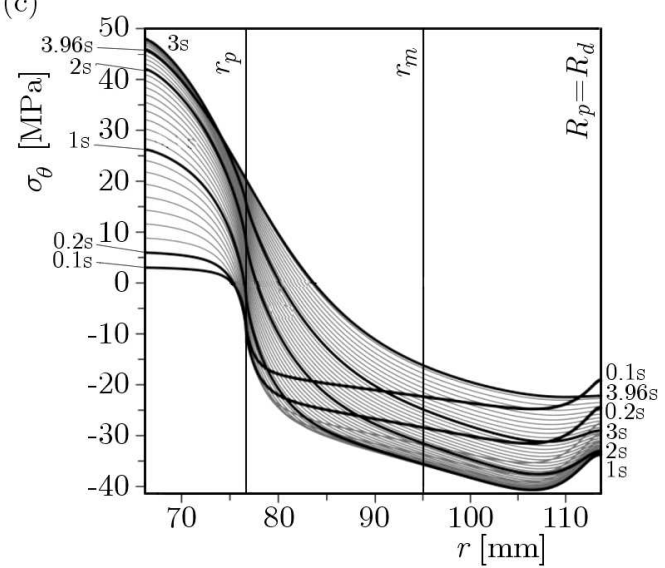

(b)

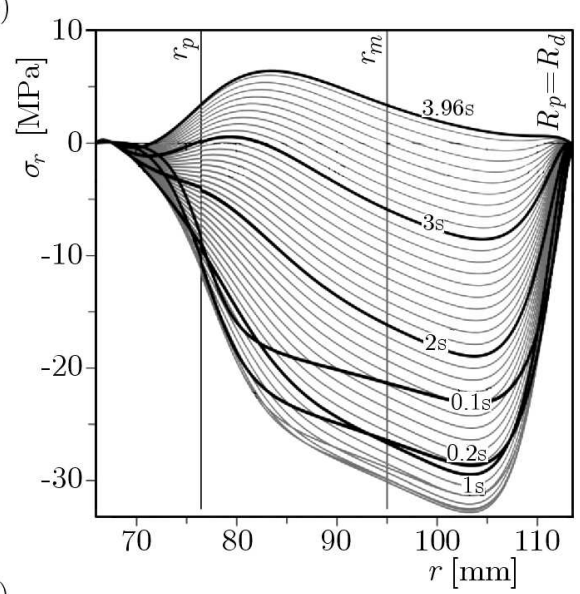

(d)

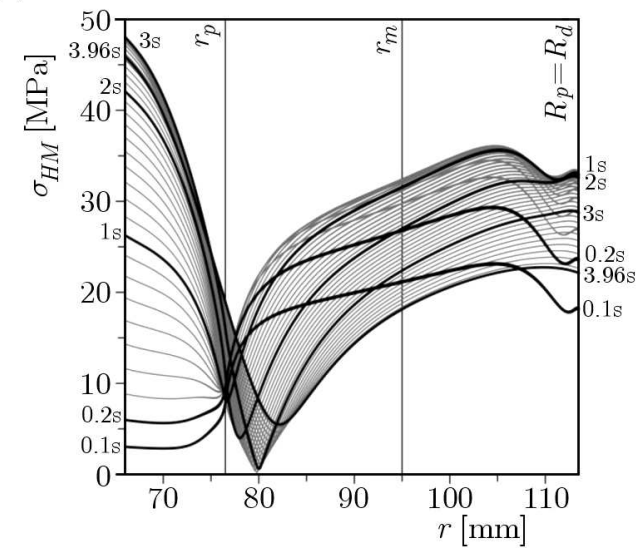

Fig. 3. Radial temperature distributions on the contact surface of the disk at selected time moments during braking (a). Radial (b), circumferential (c), and equivalent Huber-Mises (d) stress distributions on the contact surface of the disk $(z=0)$ at the selected time moments during braking

The corresponding evolutions of the radial $\sigma_{r}$, circumferential $\sigma_{\theta}$ and the equivalent Huber-Mises $\sigma_{H M}$ stresses are shown in Figs. 2bcd, respectively. The remaining components of the stress tensor $\left(\sigma_{z}\right.$ and $\left.\sigma_{r z}\right)$ are not shown due to their negligibly low values (order of $10^{-2}$ ). It can be seen that the maximum values of $\sigma_{r}, \sigma_{\theta}, \sigma_{H M}$ (during the first half of the braking time) occur on the friction surface in the range $95 \mathrm{~mm} \leqslant r \leqslant 113.5 \mathrm{~mm}$. However, after that time $(t \approx 2 \mathrm{~s})$, the highest stresses $\left(\sigma_{\theta}, \sigma_{H M}\right)$ are reached on the inner edge of disk $r_{d}=66 \mathrm{~mm}$. Maximum radial compressive (negative) stresses at $r_{p}$ and $r_{m}$ occur approximately at the same time $t \approx 0.5 \mathrm{~s}$ (Fig. 2c). Next, an apparent linear decrease in $\sigma_{r}$ to about $t \approx 3 \mathrm{~s}\left(r_{p}=76.5 \mathrm{~mm}\right)$ takes place, and the sing is changed to positive. The maximum compressive circumferential stress $\sigma_{\theta}$ in the heating area occurs slightly later (Fig. 2d). For $r_{d}=66 \mathrm{~mm}$ (inner edge of the disk), the circumferential stress throughout the entire braking process is tensile.

The temperature distributions on the friction surface $z=0$ in the radial direction $r$ for the specific time moments are shown in Fig. 3a. The temperature out of the heating area $0 \leqslant r \leqslant r_{p}$ 
increases slightly with the braking time. In the heating area $r_{p} \leqslant r \leqslant R_{p}$, the temperature increases with an increase in the distance from $z$ axis. This effect stems from the increase in the relative sliding speed which affects the heat flux density $q_{d}$, Eq. $(2.5)_{1}$.

The corresponding distributions of the stress components $\sigma_{r}, \sigma_{\theta}$ and $\sigma_{H M}$ in the radial direction $r$ are shown in Figs. 3bcd, respectively. The radial stresses $\sigma_{r}$ almost in the entire braking process are compressive (Fig. $3 \mathrm{~b}$ ). A change in the sign occurs approximately at the end of braking $(t=3.96 \mathrm{~s})$. The highest positive value is equal $\sigma_{r}=6.43 \mathrm{MPa}(r=83.45 \mathrm{~mm}$, $t=3.96 \mathrm{~s})$. The maximum negative equals $\sigma_{r}=-32.87 \mathrm{MPa}(r=103.2 \mathrm{~mm}, t=0.5 \mathrm{~s})$. It should be noted that according to the boundary conditions, Eqs. (2.9) and (2.10), the radial stress becomes zero at the outer and inner cylindrical surfaces of the disk.

The circumferential stress $\sigma_{\theta}$ is positive on the contact surface in the region free from heating $0 \leqslant r \leqslant r_{p}$ (Fig. 3c). At fixed time moments, the highest value of $\sigma_{\theta}$ in this region is obtained at the inner edge of the disk $\left(\sigma_{\theta}=48.19 \mathrm{MPa}\right.$ at $r=r_{d}=66 \mathrm{~mm}$ for $\left.t=3.2 \mathrm{~s}\right)$ and decreases withan increase in the radial distance $r$. A further increase in $r$ causes a change in the sign of $\sigma_{\theta}$ in the contact region $r_{p} \leqslant r \leqslant R_{p}$.

The maximum equivalent Huber-Mises stress $\sigma_{H M}=48.17 \mathrm{MPa}$ is reached on the inner $\left(r_{d}=66 \mathrm{~mm}\right)$ edge of the disk at the time $t=3.2 \mathrm{~s}$ (Fig. $3 \mathrm{~d}$ ). The lowest $\sigma_{H M}$ is attained near the border line of the free and heating areas $r \approx r_{p}$.

Changes in the equivalent Huber-Mises stress $\sigma_{H M}$ in the axial direction $z$ for four values of the radius $r=66,76.5,95,113.5 \mathrm{~mm}$ at the specified time moments during braking are shown in Fig. 4. Almost in all cases presented, except for the $r=76.5 \mathrm{~mm}$, the highest values of $\sigma_{H M}$ occur on the friction surface $z=0$.

(a)

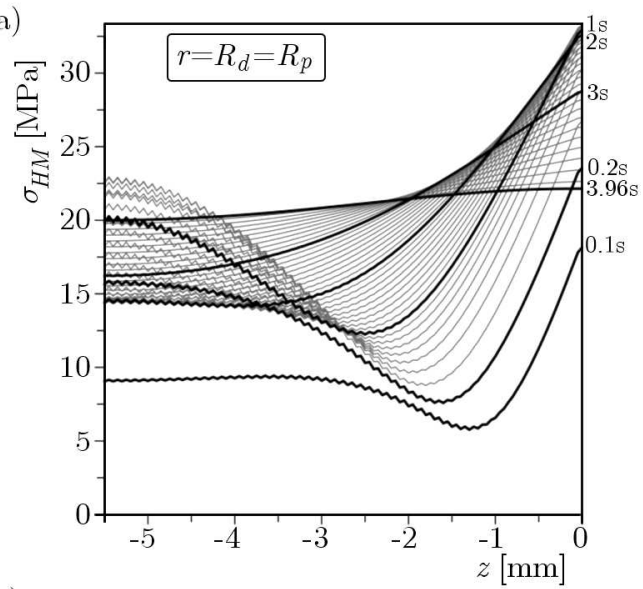

(c)

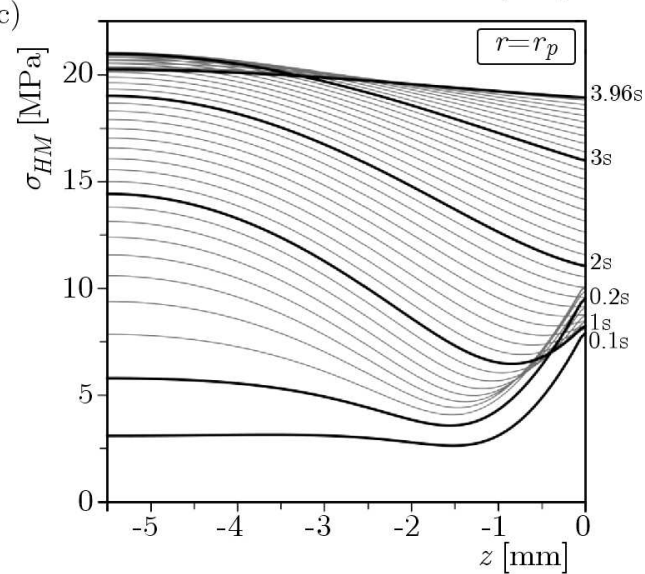

(b)

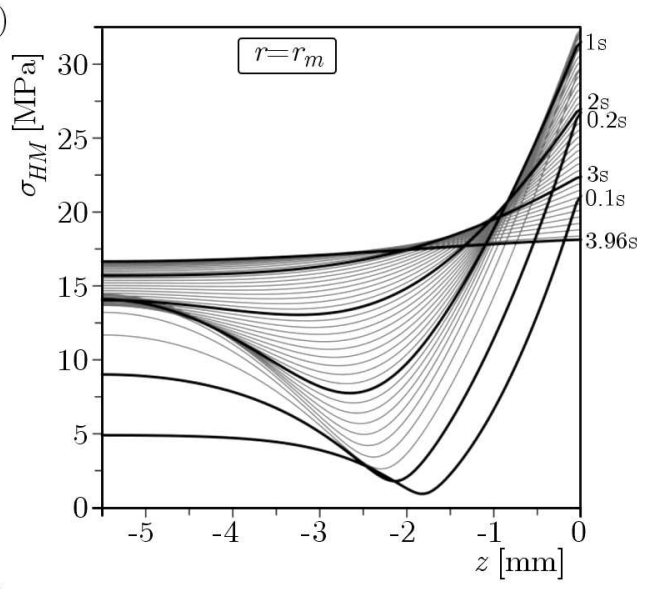

(d)

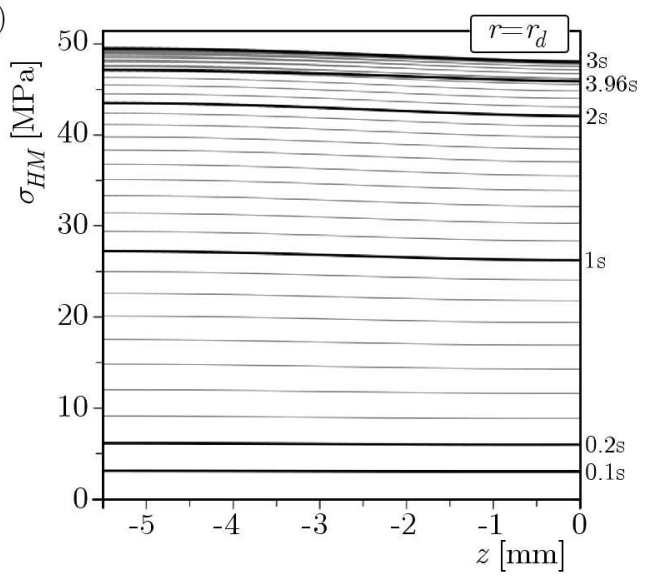

Fig. 4. Equivalent Huber-Mises stress $\sigma_{H M}$ distributions in the axial direction for $r=113.5 \mathrm{~mm}$ (a), $r=95 \mathrm{~mm}(\mathrm{~b}), r=76.5 \mathrm{~mm}(\mathrm{c})$, and $r=66 \mathrm{~mm}$ (d) at the selected time moments during braking 
The calculated isotherms in the brake disk ( $r z$ plane) at the time moments $t=0.1,0.2,1,2$, 3, 3.96 s are shown in Fig. 5a. The highest temperature values for each of the presented isotherm distributions, occurred on the friction surface $z=0$, in a short distance from the outer edge of the disk. The temperature increase for the time period $t<0.2 \mathrm{~s}$ takes place near the friction surface. With a decrease in the heat flux density $q_{d}(r, t)$, Eq. $(2.5)_{1}$, during braking, the heating weakens (a decrease in the relative slip speed) and the temperature of the disk equalizes in the axial direction $z$ (in thickness). However, the temperature distribution still remains proportional to coordinate $r$. The maximum temperature for the specified time moments equals $T=100^{\circ} \mathrm{C}$ $(t=3 \mathrm{~s})$.

(a)
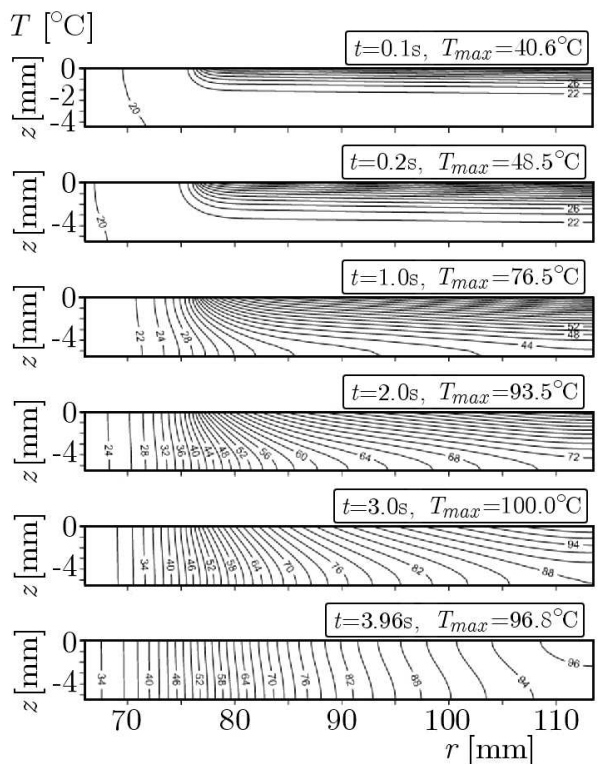

(c)

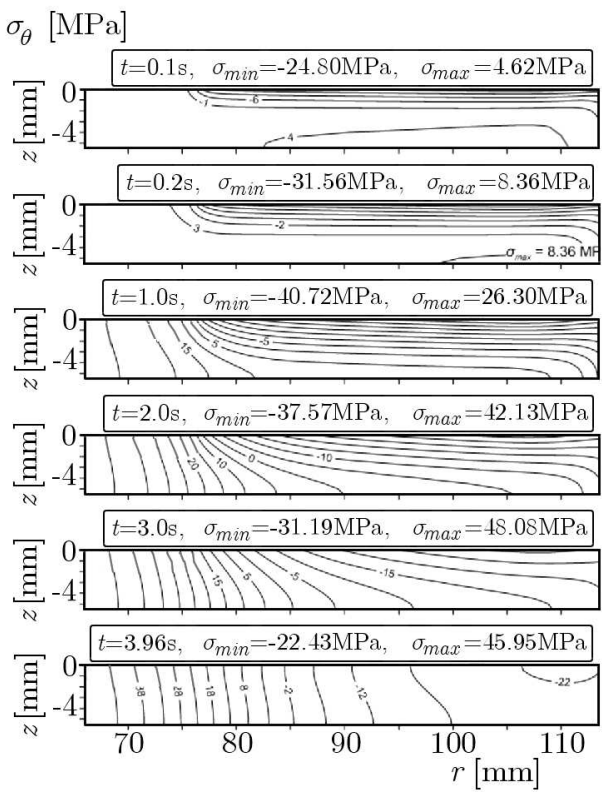

(b) $\sigma_{r}[\mathrm{MPa}]$
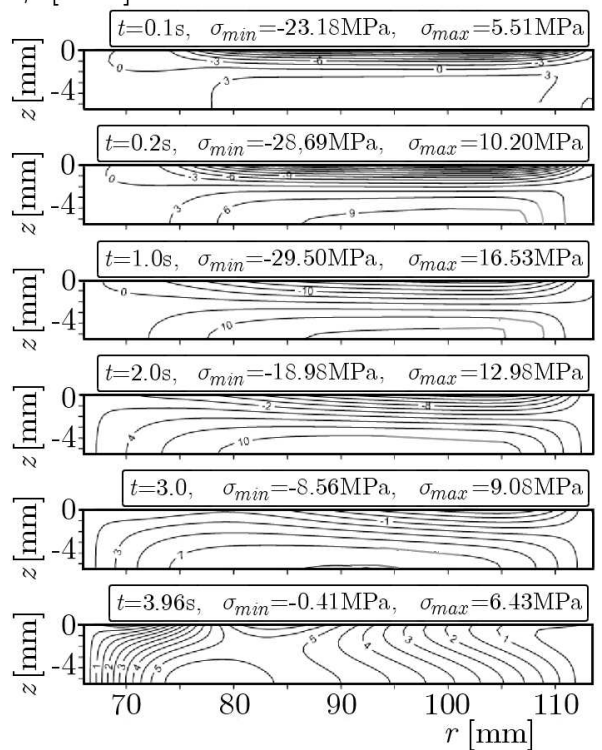

(d)
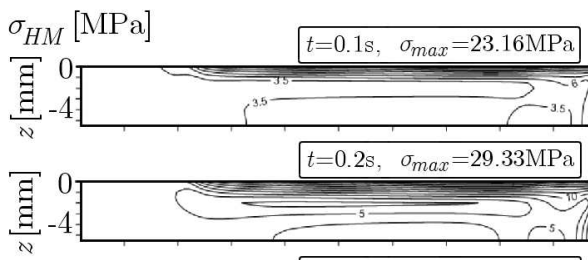

$t=1.0 \mathrm{~s}, \quad \sigma_{\max }=35.65 \mathrm{MPa}$
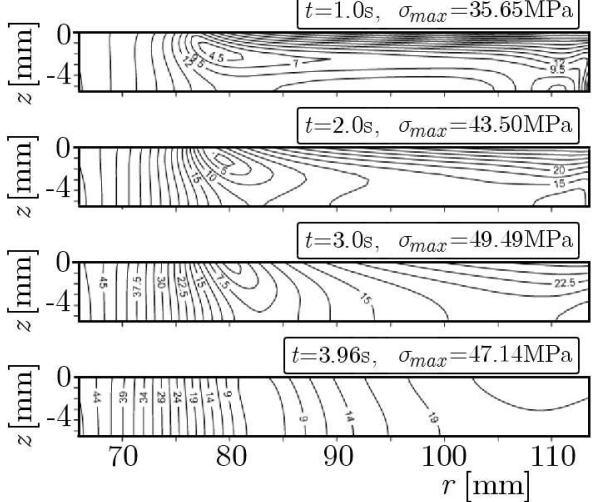

Fig. 5. Isotherms in the $r z$ plane at specific time moments during braking (a). Radial (b), circumferential (c), and equivalent Huber-Mises (d) stress isolines in the $r z$ plane at the specific time moments during braking

Based on the obtained temperature fields, the stress distributions in the disk at the previously specified time moments are calculated. Isolines of the stress tensor components $\sigma_{r}, \sigma_{\theta}$ as well 
as the equivalent Huber-Mises stress $\sigma_{H M}$ are shown in Fig. 5bcd. At the initial braking stage $\left(t<1 \mathrm{~s}\right.$ ), maximum values of stresses $\sigma_{r}, \sigma_{\theta}$ are negative (compressive) and appear on the friction surface $z=0$, near the outer edge of the disk (Fig. 5bc). The maximum tensile stresses during this period are several times lower. However, both for the positive and negative values of the stress within the entire braking process, a relationship $\sigma_{\theta}>\sigma_{r}$ is remained. After $t \approx 1 \mathrm{~s}$, the circumferential stress $\sigma_{\theta}$ (Fig. 5c), follows clear division in the disk zone between compressive (outside) and tensile stresses (inside the disk). The dividing line is fixed approximately at the distance $r_{p}$ from the axis of rotation $z$ (inner radius of the friction surface). The isolines of the Huber-Mises stress $\sigma_{H M}$ (Fig. 5d) for the braking period $t<1 \mathrm{~s}$ are similar to isotherms shown in Fig. $5 \mathrm{a}$ - the highest values of the equivalent stress $\sigma_{H M}$ are obtained on the friction surface, in a short distance from the outer edge of the disk. After that time, the area of maximum values of the equivalent stress is localized near the inner edge $\left(r<r_{p}\right)$. In the surrounding area of $r_{p}$, the minimum value of the $\sigma_{H M}$ is reached. At the stop time moment $t=3.96 \mathrm{~s}$, the isolines of the equivalent stress equalize in the thickness of the disk.

\section{Conclusions}

The axisymmetric quasi-static thermal stresses in the brake disk using the finite element method are studied. Based on the calculated results, the following conclusions are drawn:

- the radial component of the temperature gradient resulting from the thermal load $f V_{p}$ (proportional to the sliding velocity) induces the radial stress $\sigma_{r}$, which in turn affects the circumferential stress component $\sigma_{\theta}$. This agrees well with the outcomes obtained for the multi-plate clutch analyzed using FDM and FEM by Zagrodzki (1985);

- at the uniform contact pressure distribution, the highest Huber-Mises stress $\sigma_{H M}$ is reached on the inner surface of the disk; slightly lower values are attained on the friction surface $z=0$ within the outer area of the rubbing path $r_{m} \leqslant r \leqslant R_{p}$;

- for the studied problem of frictional heating of the disk during single braking of a typical passenger vehicle, the equivalent Huber-Mises stress $\sigma_{H M}$ is approximately twice lower than the yield strength of the studied material (cast iron);

- the normal circumferential stress $\sigma_{\theta}$ on the friction surface, which due to axial symmetry constitutes the principal stress, reaches maximum value. The normal axial stress component $\sigma_{z}$ is negligibly low during the considered single braking process.

The maximum equivalent Huber-Mises stress is reached on the inner cylindrical surface of the disk. In this paper. the influence of the mechanical load on the distribution of the stress in the disk is not taken into account. The study of the concurrent mechanical and temperature interactions, however of minor importance, are going to be studied further.

Acknowledgement

The present article has been financially supported by the National Science Centre in Poland (research project No. 2011/01/B/ST8/07446).

\section{References}

1. Adamowicz A., Grzes P., 2011a, Analysis of disc brake temperature distribution during single braking under non-axisymmetric load, Applied Thermal Engineering, 31, 1003-1012

2. Adamowicz A., Grzes P., 2011b, Influence of convective cooling on a disc brake temperature distribution during repetitive braking, Applied Thermal Engineering, 31, 2177-2185 
3. Adamowicz A., Grzes P., 2013, Three-dimensional FE model for calculation of temperature of a thermosensitive disc, Applied Thermal Engineering, 50, 572-581

4. Burczyński T., 1995, The Boundary Element Method in Mechanics, WNT, Warsaw

5. Chichinadze A.V., 1967, Calculation And Investigation Of External Friction During Braking (in Russian), Nauka, Moscow

6. Choi J-H., LeE I., 2003, Transient thermoelastic analysis of disk brakes in frictional contact, Journal of Thermal Stresses, 26, 223-244

7. Choi J.-H., Lee I., 2004, Finite element analysis of transient thermoelastic behaviors in disk brakes, Wear, 257, 47-58

8. Collignon M., Cristol A.-L., Dufrénoy P., Desplanques Y., Balloy D., 2013, Failure of truck brake discs: a coupled numerical-experimental approach to identifying critical thermomechanical loadings, Tribology International, 59, 114-120

9. Dufrénoy P., Weichert D., 2003, A thermomechanical model for the analysis of disc brake fracture mechanisms, Journal of Thermal Stresses, 26, 815-828

10. Evtushenko A., Matysiak S., Kutsei M., 2005, Thermal problem of friction at braking of coated body, Journal of Friction and Wear, 26, N2, 33-40

11. Gao C.H., Huang J.M., Lin X.Z., Tang X.S., 2007, Stress analysis of thermal fatigue fracture of brake disks based on thermomechanical coupling, Journal of Tribology, ASME, 129, 536-543

12. Huebner K.H., Thornton E.A., 1982, The Finite Element Method for Engineers, John Wiley \& Sons, New York

13. Mackin T.J., Noe S.C., Ball K.J., Bedell B.C., Bim-Merle D.P., Bingaman M.C., Bomleny D.M., Chemlir G.J., Clayton D.B., Evans H.A., Gau R., Hart J.L., Karney J.S., Kiple B.P., Kaluga R.C., Kung P., Law A.K., lim D., Merema R.C., Miller B.M., Miller T.R., Nielson T.J., O’Shea T.M., Olson M.T., Padilla H.A., Penner B.W., Penny C., Peterson R.P., Polidoro V.C., Raghu A., Resor B.R., Robinson B.J., Schambach D., Snyder B.D., Tom E., Tschantz R.R., Walker B.M., Wasielewski K.E., Webb T.R., Wise S.A., Yang R.S., Zimmerman R. S., 2002, Thermal cracking in disc brakes, Engineering Failure Analysis, 9, 63-76

14. Matysiak S. J., Yevtushenko A., Kuciej M., 2007, Temperature field in the process of braking of a massive body with composite coating, Materials Science, 43, N1, 62-69

15. MSC. Nastran Thermal Analysis User's Guide, 2002

16. Noda N., Hetnarski R.B., Tanigawa Y., 2003, Thermal Stresses, Taylor \& Francis, New York

17. Sakamoto H., Hirakawa K., 2005, Fracture analysis and material improvement of brake discs, JSME International Journal, Series A - Solid Mechanics, 48, 458-464

18. ScieszkA S.F., Zolnierz M., 2014, Experimental and numerical investigations of thermomechanical instability of the industrial disc brakes, Proceedings of the Institution of Mechanical Engineers, Part J: Journal of Engineering Tribology, 228, 5. 567-576

19. Talati F., Jalalifar S., 2009, Analysis of heat conduction in a disk brake system, Heat Mass Transfer, 45, 1047-1059

20. Yevtushenko A.A., Grzes P., 2010, FEM-modeling of the frictional heating phenomenon in the pad/disc tribosystem (a review), Numerical Heat Transfer, Part A - Applications, 58, 207-226

21. Yevtushenko A., Grzes P., 2011, Finite element analysis of heat partition in a pad/disc brake system, Numerical Heat Transfer, Part A - Applications, 59, 521-542

22. Yevtushenko A.A., Grzes P., 2012, Axisymmetric finite element model for the calculation of temperature at braking for thermosensitive materials of a pad and a disc, Numerical Heat Transfer, Part A - Applications, 62, 211-230 
23. Yevtushenko A.A., Grzes P., Adamowicz A., 2014, Numerical analysis of thermal stresses in disk brakes and clutches (a review),Numerical Heat Transfer, Part A - Applications, ID: 923221, DOI: $10.1080 / 10407782.2014 .923221$

24. Zagrodzki P., 1985, Numerical analysis of temperature fields and thermal stresses in the friction discs of a multidisc wet clutch, Wear, 101, 255-271

25. ZAGrodzki P., 1990, Analysis of thermomechanical phenomena in multidisc clutches and brakes, Wear, 140, 291-308

26. Zhao S., Hilmas G., Dharani L.R., 2008, Behavior of a composite multidisk clutch subjected to mechanical and frictionally excited thermal load, Wear, 264, 1059-1068

27. Zienkiewicz O.C., Taylor R.L., Zhu J.Z., 2005, The Finite Element Method: Its Basis And Fundamentals, Elsevier Butterworth-Heinemann, Amsterdam

Manuscript received October 1, 2014; accepted for print November 2, 2014 